\title{
Development of Mycobacterium tuberculosis attenuated strains as live vaccine candidates for tuberculosis
}

\author{
Anne Villela*, Valnês Rodrigues-Junior, Luiz Augusto Basso, Diógenes Santos \\ From 5th Congress of the Brazilian Biotechnology Society (SBBIOTEC) \\ Florianópolis, Brazil. 10-14 November 2013
}

Tuberculosis (TB) is an infectious disease caused mainly by the bacillus Mycobacterium tuberculosis. The World Health Organization estimates that 8.6 million new TB cases occurred in 2012, resulting in 1.3 million deaths [1]. $\mathrm{TB}$ is the second leading cause of death caused by an infectious disease worldwide after immunodeficiency virus (HIV). Despite the availability of an effective chemotherapy and a moderately protective Bacille-Calmette-Guérin (BCG) vaccine, TB remains a major global health problem [1]. BCG provides efficient protection against TB in newborns, but its efficacy against the establishment of latent or pulmonary $\mathrm{TB}$ in adults is highly variable. The variability of BCG protection in adults might be related to the absence of more than a hundred genes when compared with the Mycobacterium bovis pathogenic strain. Among the missing genes in BCG is the RD1 region which encodes potent antigens and virulence factors [2]. Thus, there is an urgent need for the emergence of new prophylactic strategies to decrease TB incidence worldwide. The development of genetic tools to manipulate mycobacteria and the completion of $M$. tuberculosis genome sequencing have been contributing to a better understanding of genes involved in TB virulence and pathogenesis, and consequently to the emergence of novel vaccine candidates.

Two major strategies have been used to develop new vaccine candidates against TB: (i) substitution of BCG in which an improved version of BCG or a new attenuated live $M$. tuberculosis vaccine would have a higher efficiency than BCG and replace it as a prime vaccine; and (ii) a prime-boost strategy in which BCG continues to be given to neonates, and a new vaccine is given as a booster dose

\footnotetext{
Centro de Pesquisas em Biologia Molecular e Funcional (CPBMF), Instituto Nacional de Ciência e Tecnologia em Tuberculose (INCT-TB), PUCRS. Av. Ipiranga, 6681 - TECNOPUC - Prédio 92A Partenon - Porto Alegre, CEP 90619-900, Brazil
}

to extend the protection and efficacy [1]. Following these approaches, numerous vaccine candidates against $\mathrm{TB}$ are currently in preclinical and clinical trials, including recombinant BCGs, attenuated $M$. tuberculosis strains, recombinant viral-vectored platforms, protein/adjuvants combinations and mycobacterial extracts [1]. The strategy to develop novel vaccines based on the construction of rationally attenuated $M$. tuberculosis strains holds the advantage of potentially eliciting a more sustained protective immune response than viral vectored and recombinant protein candidates.

The successful isolation of allelic exchange mutants of M. tuberculosis is dependent on the ability of the genetic tools to enable the efficient detection and selection of mutants among the total population of transformants [3]. Suicide and conditionally-replicating delivery plasmids that combines both selectable and counter-selectable markers, reporter gene, and a mycobacterial thermosensitive origin of replication have been widely used to demonstrate attenuation of M. tuberculosis mutant strains [3].

The first step towards the development of a new live vaccine candidate against TB is the evaluation of mutant strain attenuation followed by the investigation of its ability to generate an immune response. Live vaccine candidates have to mimic natural infection as closely as possible without causing disease, in which, a right balance between attenuation and immunogenicity has to be reached, since over attenuated bacteria may not produce in vivo some key antigens for the induction of a protective immunity [4].

An efficient strategy to rationally attenuate $M$. tuberculosis consists on the construction of double-deletion mutant of $M$. tuberculosis in the panC and panD genes, both involved in the de novo biosynthesis of pantothenate. Pantothenic acid (vitamin B5) is an essential molecule required for the synthesis of coenzyme A and acyl carrier 
protein $(\mathrm{ACP})$, two important molecules in fatty acid metabolism and other metabolic reactions [5]. The double-deletion mutant resulted in an auxotrophic and attenuated strain which conferred protection in mice challenged with virulent $M$. tuberculosis [5]. In attempts to further enhance the safety of this live attenuated $M$. tuberculosis vaccine candidate, two other mutant strains were constructed combining panCD deletion with either lys $A$ or leuD deletions, involved in lysine and leucine biosynthesis, respectively. The $\mathrm{mc}^{2} 6020$ strain, constructed by the inactivation of the panCD and lysA genes, is strictly auxotrophic for pantothenate and lysine, severely attenuated and capable of inducing protective responses against an aerosolized M. tuberculosis challenge in both immunocompetent and immunocompromised mice [6]. The strain constructed by inactivation of the panCD and leuD genes was shown to confer long-term protection against challenge with virulent $M$. tuberculosis in guinea pig model which is equivalent to that afforded by BCG [7]. Simian immunodeficiency virus (SIV)-positive and SIV-negative Rhesus macaques were immunized with $p a n C D$ and leuD mutant strain, and safety studies, clinical, hematological and bacteriological monitoring were carried out and revealed no vaccine-associated adverse effects [7].

Another successful approach employed to rationally attenuate $M$. tuberculosis combines deletions in secA2 and lys $A$ genes. The $\sec A 2$ gene encodes a component of a virulence-associated bacterial protein secretion system involved in inhibiting the host immune system and consequently promoting the $M$. tuberculosis survival within the host. Thus, secA2 mutant was shown to increase both host cell apoptosis and priming of antigen-specific $\mathrm{CD} 8^{+} \mathrm{T}$ cells in vivo. The secA2 and lys $A$ double-mutant strain retained the effects obtained by sec $A 2$ single mutation, but with an improved safety profile in immunosuppressed mice [8].

Recently, the first live-attenuated M. tuberculosis-based vaccine, MTBVAC, entered clinical trials. MTBVAC contains in two independent deletions without antibiotic-resistance markers in the genes phoP, coding for a transcription factor key for the regulation of $M$. tuberculosis virulence, and fadD26, coding for one of the major mycobacterial virulence factors [9]. First, a mutant strain containing a single mutation in $p h o P$ gene was constructed and showed a high degree of safety, improved immunogenicity and protective efficacy compared to BCG in several animal models, from mice to non-human primates. Then, a second independent mutation deleting $f a d D 26$ gene was introduced to obtain MTBVAC which was shown to be functionally and phenotypically comparable to its prototype after rigorous preclinical safety and biodistribution experiments [9]. This vaccine candidate was genetically engineered to fulfill the Geneva consensus requirements for Phase I clinical trials of live mycobacterial vaccines candidates which demands improved protective efficacy and safety potential relative to BCG, and two non-reverting independent mutations without antibiotic resistance markers [10].

Overall, these multiple deletion mutants demonstrated the feasibility to obtain rationally attenuated $M$. tuberculosis strains, which so far were shown to be safe without compromising their ability to provide protective immunity, making them viable live vaccines candidates against $\mathrm{TB}$.

Published: 1 October 2014

\section{References}

1. World Health Organization, Global Tuberculosis Report. 2013 [http:// www.who.int/tb/publications/global_report/en/].

2. Martin C, Walker B: Attenuated strains of Mycobacterium tuberculosis complex for laboratory and clinical use. Tuberculosis 2008, 88:371-374.

3. Jackson M, Camacho LR, Gicquel B, Guilhot C: Gene replacement and transposon delivery using the negative selection marker sacB. In Mycobacterium tuberculosis protocols. Totowa: Humana Press; Parish T and Stocker NG 2001:59-75.

4. Jackson M, Phalen SW, Lagranderie M, Ensergueix D, Chavarot P, Marchal G, McMurray DN, Gicquel B, Guilhot C: Persistence and protective efficacy of a Mycobacterium tuberculosis auxotroph vaccine. Infect Immun 1999, 67:2867-2873.

5. Sambandamurthy VK, Wang $X$, Chen B, Russell RG, Derrick S, Collins FM, Morris SL, Jacobs WR Jr: A pantothenate auxotroph of Mycobacterium tuberculosis is highly attenuated and protects mice against tuberculosis. Nat Med 2002, 8:1171-1174

6. Sambandamurthy VK, Derrick SC, Jalapathy KV, Chen B, Russell RG, Morris SL, Jacobs WR Jr: Long-term protection against tuberculosis following vaccination with a severely attenuated double lysine and pantothenate auxotroph of Mycobacterium tuberculosis. Infect Immun 2005, 73:1196-1203.

7. Sampson SL, Mansfield KG, Carville A, Magee DM, Quitugua T, Howerth EW, Bloom BR, Hondalus MK: Extended safety and efficacy studies of a live attenuated double leucine and pantothenate auxotroph of Mycobacterium tuberculosis as a vaccine candidate. Vaccine 2011, 29:4839-4847.

8. Hinchey J, Jeon BY, Alley H, Chen B, Goldberg M, Derrick S, Morris S, Jacobs WR Jr, Porcelli SA, Lee S: Lysine auxotrophy combined with deletion of the SecA2 gene results in a safe and highly immunogenic candidate live attenuated vaccine for tuberculosis. PLoS One 2011, 6:e15857.

9. Arbues A, Aguilo JI, Gonzalo-Asensio J, Marinova D, Uranga S, Puentes E, Fernandez C, Parra A, Cardona PJ, Vilaplana C, Ausina V, Williams A, Clark S, Malaga W, Guilhot C, Gicquel B, Martin C: Construction, characterization and preclinical evaluation of MTBVAC, the first live-attenuated M. tuberculosis-based vaccine to enter clinical trials. Vaccine 2013, 31:4867-4873.

10. Walker KB, Brennan MJ, Ho MM, Eskola J, Thiry G, Sadoff J, Dobbelaer R, Grode L, Liu MA, Fruth U, Lambert PH: The second Geneva Consensus: Recommendations for novel live TB vaccines. Vaccine 2010, 28:2259-2270.

doi:10.1186/1753-6561-8-S4-05

Cite this article as: Villela et al:: Development of Mycobacterium tuberculosis attenuated strains as live vaccine candidates for tuberculosis. BMC Proceedings 2014 8(Suppl 4):O5. 